\title{
Are Inflammatory and Coagulation Biomarkers Related to Sleep Characteristics in Mid-Life Women?: Study of Women's Health Across the Nation Sleep Study
}

Karen A. Matthews, PhD'1,4 Huiyong Zheng, PhD²; Howard M. Kravitz, DO, MPH³; MaryFran Sowers, PhD²; Joyce T. Bromberger, PhD 1,4; Daniel J. Buysse, MD; Jane F. Owens, DrPH${ }^{1}$; Mark Sanders, MD ${ }^{5}$; Martica Hall, $\mathrm{PhD}^{1}$

${ }^{1}$ Department of Psychiatry, University of Pittsburgh, Pittsburgh, PA; ${ }^{2}$ Department of Epidemiology, University of Michigan, Ann Arbor, MI; ${ }^{3}$ Departments of Psychiatry and Preventive Medicine, Rush University Medical Center, Chicago, IL; ${ }^{4}$ Department of Epidemiology, University of Pittsburgh, Pittsburgh, PA; ${ }^{5}$ Department of Medicine, University of Pittsburgh, Pittsburgh, PA (retired)

\begin{abstract}
Study Objectives: Inflammation and pro-coagulation biomarkers may be a link between sleep characteristics and risk for cardiometabolic disorders. We tested the hypothesis that worse sleep characteristics would be associated with C-reactive protein (CRP), fibrinogen, factor VIIc, and plasminogen activator inhibitor (PAI)-1 in a multi-ethnic subsample of mid-life women enrolled in the Study of Women's Health across the Nation.

Design: Cross-sectional.

Measurements and Results: African American, Chinese, and Caucasian women $(\mathrm{N}=340)$ participated in 3 days of in-home polysomnographic (PSG) monitoring and had measures of inflammation and coagulation. Regression analyses revealed that each of the biomarkers were associated with indicators of sleep disordered breathing after adjusting for age, duration between sleep study and blood draw, site, menopausal status, ethnicity, residualized body mass index, smoking status, and medications that affect sleep or biomarkers. Among African American women, those who had higher levels of CRP had shorter PSG-sleep duration and those who had higher levels of fibrinogen had less efficient sleep in multivariate models. Conclusions: These results suggest that inflammation and pro-coagulation processes may be an important pathway connecting sleep disordered breathing and cardiometabolic disorders in women of these ethnic groups and that inflammation may be a particularly important pathway in African Americans.
\end{abstract}

Keywords: Inflammation, coagulation, C-reactive protein, fibrinogen, PAI-1, race, women, sleep disordered breathing, sleep duration, sleep efficiency

Citation: Matthews KA; Zheng H; Kravitz HM; Sowers M; Bromberger JT; Buysse DJ; Owens JF; Sanders M; Hall M. Are inflammatory and coagulation biomarkers related to sleep characteristics in mid-life women?: Study of Women's Health Across the Nation Sleep Study. SLEEP 2010;33(12):1649-1655.

SLEEP DISORDERED BREATHING, SELF-REPORTED SLEEP DURATION, AND PERHAPS INSOMNIA ARE RELATED TO RISK FOR CARDIOVASCULAR DISEASE (CVD), type 2 diabetes, and obesity. ${ }^{1-5}$ Inflammatory and coagulation biomarkers are also related to risk for these conditions. $^{6-8}$ It has been suggested that activation of inflammatory and pro-coagulation pathways mediates the relationships between sleep characteristics and cardiometabolic health..$^{9,10}$ The purpose of the present investigation is to evaluate the cross-sectional association between sleep characteristics and circulating inflammatory and coagulation markers in an ethnically diverse sample of middle-aged women.

Inflammation, blood coagulation, and immune responses are tightly linked and interdependent processes. Chronic low level inflammation contributes to coronary heart disease (CHD) through atherogenesis, plaque progression, and eventually plaque erosion and rupture. ${ }^{11}$ Moreover, atherosclerosis and vascular damage are pro-inflammatory, thereby establishing a vicious cycle. Increased activity through pro-coagulation pathways and diminished fibrinolysis has also been implicated in the pathophysiology of $\mathrm{CHD},{ }^{12,13}$ although it has been ar-

Submitted for publication December, 2009

Submitted in final revised form July, 2010

Accepted for publication July, 2010

Address correspondence to: Karen A. Matthews, PhD, University of Pittsburgh, Department of Psychiatry, 3811 O'Hara Street, Pittsburgh, PA 15213; Tel: (412) 648-7158; Fax: (412) 648-7160; E-mail: matthewska@ upmc.edu gued that a pre-existing hypercoagulable status is not causal. ${ }^{14}$ Rather atherosclerosis and vascular damage may cause changes in coagulation, which can feed back to inflammation. Marked acute and chronic inflammatory responses occur with infection, smoking, insulin resistance, and adiposity. Markers of inflammation include C-reactive protein (CRP), fibrinogen, and plasminogen activator inhibitor (PAI)-1. Fibrinogen also leads to increased pro-coagulant activity and plasma viscosity, factor VIIc to increased pro-coagulant activity, and both fibrinogen and PAI-1 to decreased fibrinolysis.

In the present study, we evaluated the associations of biomarkers of inflammation and coagulation (CRP, fibrinogen, factor VIIc, and PAI-1) with sleep measured for 3 nights by in-home PSG studies as well as by self-report in community samples of mid-life Chinese, African American, and Caucasian women. We hypothesized that impaired sleep characteristics (short sleep duration, low continuity, low percentage of delta sleep, sleep disordered breathing, and poor sleep quality) would be associated with elevated biomarkers. We have previously reported substantial ethnic differences in sleep in our sample ${ }^{15}$ and in the biomarkers in the larger study from which our participants were drawn. ${ }^{16}$ Therefore, we also explored ethnic differences in the pattern of results. Thus, this study adds to the literature in several ways: It assessed both sleep and biomarkers of inflammation and coagulation in a comprehensive manner. It assessed sleep in the women's home increasing the ecological validity of the sleep assessments, relative to clinic-based assessments. It was based on community as opposed to clinic samples with known sleep disorders or to exceptionally healthy individuals enrolled in sleep deprivation experiments. Partici- 
pants were in the menopausal transition, a time of increased sleep complaints. Finally, we tested for ethnic differences in the pattern of results, which, to our knowledge, has not been evaluated in prior research on connections between sleep and inflammation and coagulation.

\section{METHODS}

\section{Participants}

Participants were enrolled in the Study of Women's Health Across the Nation (SWAN) ancillary Sleep Study. SWAN is a community-based study of midlife aging in women. ${ }^{17}$ Eligibility criteria for the longitudinal cohort were ages 42-52 years, having an intact uterus, having at least one menstrual period and not using exogenous hormones (birth control or hormone therapy) in the 3 months prior to the baseline interview, and having self-identified with the site's designated race/ethnic groups. The institutional review boards at all participating sites approved the study protocol.

The objective of the SWAN Sleep Study was to examine changes in sleep during the menopausal transition and their associations with health and functioning. It enrolled a cohort of 370 Caucasian, African American, and Chinese participants from 4 of the 7 study sites located in Chicago, IL; Detroit area, MI; Oakland, CA; and Pittsburgh, PA. Efforts were focused on recruiting women in the menopausal transition, resulting in 328 pre- and peri-menopausal women, and 42 early postmenopausal women not using hormone therapy at the time of recruitment into the sleep study. Exclusions for the Sleep Study were: diagnosed sleep disorders; current chemotherapy or radiation; current oral corticosteroid use; regular night shift work; regular consumption of $\geq 4$ alcoholic drinks/day; and noncompliance with Core SWAN procedures (missed $>50 \%$ of annual visits, refused annual visit blood draw). Women on medications that might affect sleep or inflammation were not excluded; statistical analyses controlled for use of these medications (see below). Of the 370 participants, 366 had PSG data. Twenty-six women at the Chicago site did not have biomarkers available because of local financial constraints; so a total of 340 were included in the analyses. Informed consent for the SWAN Sleep Study was obtained in accordance with approved protocols and guidelines of the institutional review board at each participating institution. Participants received $\$ 250$ for their participation in all aspects of the protocol. The payment included $\$ 150$ for completing the PSG assessments themselves.

\section{Protocol and Assessments}

The SWAN Sleep Study protocol was conducted across participants' entire menstrual cycle or 35 days, whichever was shorter. The duration restriction was in place because some women had long intervals between cycles or were postmenopausal. After women agreed to participate in the sleep study, the pre- and peri-menopausal women identified the expected date of their next menses for study planning purposes. They were asked to contact the clinic to schedule their PSG measures on the first day of their next menses. Unattended PSG sleep studies were conducted in participants' homes on the first 3 nights of the protocol, usually on days 3 to 6 of their menses, i.e., follicular phase, if they were menstruating. SWAN Sleep Study staff vis- ited participants in their homes on each night of PSG studies to apply electrodes and calibrate monitors when participants were already in their bedclothes. Participants were asked to sleep in their own beds and maintain their habitual sleep and wake times, as determined by self-report (rather than go to bed immediately after departure of staff). Upon rising in the morning, participants removed the PSG equipment and turned off the recorder.

\section{Sleep}

Measures included subjective sleep quality and indices of PSG-assessed sleep duration, continuity, depth and sleep disordered breathing. The 19-item self-report Pittsburgh Sleep Quality Index (PSQI) ${ }^{18}$ was measured at the beginning of the study. A summary score was computed with higher scores representing more severe sleep complaints. Because some studies reported associations with self-reported sleep duration, even in the absence of associations with objective sleep duration, we also examined the responses to the PSQI question regarding actual sleep time in the last month. This item correlated moderately with PSG total duration, $\mathrm{r}=0.32, \mathrm{P}<0.0001$. PSG sleep data were collected with Vitaport-3 (TEMEC VP3) ambulatory monitors. Signals collected on each study night included bilateral central referential EEG channels $\left(\mathrm{C}_{3}\right.$ and $\mathrm{C}_{4}$, referenced to $\mathrm{A}_{1}-\mathrm{A}_{2}$ ), electro-oculogram (EOG), submentalis electromyogram (EMG), and electrocardiogram (EKG). Additional signals were collected on the first night of sleep studies for the assessment of sleep disordered breathing (SDB; nasal pressure and oral-nasal thermistors, pulse oximetry, and abdominal and thoracic excursion, as measured by inductance plethysmography to reflect ventilatory effort). Quality assurance assessments, scoring, and processing of all sleep study records were performed at the University of Pittsburgh Neuroscience-Clinical and Translational Research Center (N-CTRC). Visual sleep stage scoring was conducted by trained PSG technologists with established reliability (intraclass correlation coefficients for wake, NREM, and REM were each > 0.90), who were blind to participant characteristics. Sleep was scored in 20-sec epochs using standard scoring criteria. ${ }^{19,20}$

Measures of sleep duration, continuity (efficiency and wake after sleep onset or WASO), and depth (stage 3-4) were based on averages of sleep study nights 2 and 3; whereas sleep disordered breathing was assessed on night 1 only. (AASM criteria for sleep stages were not published at the time that scoring was completed.) Total sleep time (TST; sleep duration) was calculated as total minutes of any stage of sleep from sleep onset to morning awakening converted into hours. TST/time spent in bed x 100 was used to quantify sleep efficiency. Sleep-disordered breathing was quantified by the apnea-hypopnea index (AHI; number of apneas + number of hypopneas/TST) ${ }^{19}$; desaturation event frequency (DEF; number of oxyhemoglobin desaturations $\geq 4 \%$ divided by $\mathrm{TST}$ ) and percent $\mathrm{TST} \leq 90 \%$ saturation. WASO and AHI were log transformed prior to analyses because of their distributions.

\section{Covariates}

Body mass index was measured in clinic as part of the annual SWAN core examination. Race/ethnicity was established at the first visit by self-identification (non-Hispanic Caucasian, Chinese, or African American). Participants were categorized as 
premenopausal, early perimenopausal (menses in last 3 months but irregular), late perimenopausal (no menses for 3-11 months), and postmenopausal (no menses for at least 12 months or hysterectomy or bilateral oophorectomy), based on self-reported menstrual bleeding patterns. Health behaviors were assessed by daily diary reports of smoking (any nicotine use was coded as "yes"). Medication use, recorded at Sleep Study protocol inception, was coded according to the World Health Organization ATC classification (http://www.whocc.no/atcddd). For the present report, medication use was based on daily diary records maintained by study participants and initiated at the time of the sleep study. Medications that affect sleep were considered to be those products associated with the following ATC classification codes: N02A (opioids), N03A (antiepileptics), N05B (anxiolytics), N05C (hypnotics and sedatives), N06A (antidepressants), and R06A (antihistamines). Medications that affect inflammation and coagulation were considered to be those products associated with the following ATC classification codes: A10A or $\mathrm{B}$ (insulin); BO1A (antithrombotics); CO1A, CO2D, CO3A-D and $\mathrm{X}, \mathrm{CO} 7 \mathrm{~A}, \mathrm{X}, \mathrm{CO} 8 \mathrm{C}, \mathrm{D}, \mathrm{C} 09 \mathrm{C}, \mathrm{C} 10 \mathrm{~A}$ (cardiovascular); HO2A, HO3A, B (steroids); M01A (anti-inflammatory); R03A, $\mathrm{B}$, and D (adrenergics), RO5C,D, and X (cold medicines). Medication use was dichotomized as "present" or "absent" for sleep and biomarkers separately. Use of hormone therapy at time of blood draw and site also served as covariates.

\section{Markers of Inflammation and Coagulation}

An annual fasting blood draw was targeted to the early follicular phase of the menstrual cycle in menstruating women and prior to 10:00 a.m. in an effort to provide a standardized hormonal milieu. All samples were maintained at $4{ }^{\circ} \mathrm{C}$ until separated and then were frozen at $-80^{\circ} \mathrm{C}$ and shipped on dry ice to a central laboratory (Medical Research Laboratories, Highland Heights, KY, USA), which is certified by the National Heart Lung and Blood Institute, Centers for Disease Control Lipid Standardization Part III program. Fibrinogen and factor VIIc were measured in frozen citrated plasma using a clot-based turbidometric detection system, with the Factor VII assay using Factor VII deficient plasma in preparing the standard curve. PAI-1 was measured using a solid phased monoclonal antibody and a second enzyme-labeled goat antiserum for detection (American Diagnostica, Greenwich, CT). CRP was measured using an ultra-sensitive rate immunonephelometry (DadeBehring, Marburg, Germany). Inflammatory and coagulation measures were taken from the annual SWAN examination prior to but closest in time to the SWAN Sleep data collection. In this regard, high test-retest correlations were apparent between measures at the SWAN core annual examinations prior to and following the SWAN Sleep Study: Pearson $r=0.83$ for PAI-1, 0.88 for factor VIIc, 0.93 for fibrinogen, and 0.96 for CRP. CRP data for 44 women with values $>10$ were removed because they may have reflected infection. All values were natural log transformed prior to analyses because of their distribution.

\section{Data Analysis}

Kruskal-Wallis tests were used to compare the mean rank of markers according to race/ethnicity and $\chi^{2}$ tests were used to compare the proportions of categorical independent variables and covariates. Because adiposity is a source of inflamma- tion, we calculated BMI residuals using linear regressions on $\log$ BMI with the predictors of each hemostatic/inflammatory marker taken individually. We then calculated initial linear regression models on each sleep measure with each of the inflammatory and coagulation measures, adjusted for age at the sleep study and time duration elapsed from the measurements of biomarkers to sleep study (Model 1). Because only 11 women had PSG-measured sleep duration $>8 \mathrm{~h}$, we did not examine the curvilinear relationship between sleep duration and biomarkers. Fully adjusted Model 2 controlled for age, duration between measures, residualized BMI, medications that affect sleep or the hemostatic/inflammatory markers, hormone therapy use, smoking status, menopausal status, site, and ethnicity. P-values from 2-sided tests at a value of $\mathrm{P}<0.01$ were considered statistically significant because we measured multiple indicators of 5 sleep dimensions: duration, continuity, depth, sleep disordered breathing, and sleep quality. These analyses were followed by tests for interactions between the inflammatory/hemostatic marker and ethnicity in multivariate models. Significant interactions were further evaluated by analyses stratified by ethnicity. Because these analyses were exploratory, we report the results for analyses with interactions terms significant at $\mathrm{P}<0.05$ (2-sided). SAS 9.1 and Macro facilities (SAS Institute, Cary, NC) were used to perform the statistical analyses.

\section{RESULTS}

\section{Sample Description}

Participants in the SWAN Sleep Study were 125 African American, 156 Caucasian, and 59 Chinese (Table 1). About $40 \%$ were obese but few were current smokers, and only 4 had been diagnosed by a physician/health care provider with stroke or heart disease. Fifty-six percent were taking medications that could affect biomarkers of inflammation or coagulation, 15\% were taking antidepressant medication; and about a quarter of the women were taking medications that could affect sleep at the time of the SWAN Sleep Study. Overall, women slept on average $6.25 \mathrm{~h}$ measured by PSG, with about $0.75 \mathrm{~h}$ WASO, and a small percent of stage 3-4 sleep (Table 2). About 20\% had AHI scores $\geq 15$ events per hour. Women reported somewhat elevated PSQI scores, relative to previously published values for these scales. Substantial ethnic differences were found in the sociodemographic, inflammatory, and coagulation measures, and sleep characteristics. Consistent with reports from the full SWAN cohort, ${ }^{16}$ African American women had elevated CRP, fibrinogen levels, and BMI compared to the other groups (Table 1). As reported elsewhere, in the present sample, African American women slept less and had poorer sleep continuity and sleep quality than whites or Chinese. ${ }^{15}$

The inflammatory and coagulations measures were interrelated: CRP was associated with fibrinogen, factor VIIc, and PAI-1 $(\mathrm{r}=0.48,0.26$, and 0.37 , respectively). Fibrinogen was associated with factor VIIc and PAI- $1(\mathrm{r}=0.26$ and 0.20 , respectively), and factor VIIC and PAI-1 were associated $(\mathrm{r}=0.15)$.

\section{Associations between Sleep Characteristics and Inflammatory/ Coagulation Measures}

Higher CRP levels were associated with lower sleep efficiency, higher sleep disorder breathing measures (higher AHI, 
Table 1-Sample characteristics at sleep study or visit prior to sleep study

No. (\% of total)

Median (IQR) age (years)

Median (IQR) C-reactive protein (CRP) $\mathrm{mg} / \mathrm{dL}$

No. (\%) CRP $\geq 3$

Median (IQR) fibrinogen $\mathrm{mg} / \mathrm{dL}$

Median (IQR) \% factor VII-c mg/dL

Median (IQR) plasminogen activator inhibitor -1

(PAl-1) $\mathrm{mg} / \mathrm{dL}$

Median (IQR) body mass index (BMI) $\mathrm{kg} / \mathrm{m}^{2}$

No. (\%) BMI $\geq 30$

No. $(\%)$ current smoker

No. (\%) stroke/heart

No. (\%) medications affecting hemostatic factors

No. (\%) antidepressant medication

No. (\%) hormone therapy

Total Sample
340
$52(3)$
$2.0(3.8)$
$133(39.4)$
$273(78)$
$119(38)$
$13.8(19.0)$

$28.1(10.9)$

$135(41.0)$

$31(9.3)$

$4(1.2)$

$186(55.7)$

50 (15.0)

$19(5.6)$
African

Americans

125 (36.8)

52 (4)

3.4 (6.8)

$68(54.4)$

292 (78)

115 (37)

14.8 (19.3)

32.6 (10.5)

76 (64.4)

24 (20.2)

$1(0.8)$

76 (62.8)

14 (11.6)

3 (2.4)

Caucasians
$156(45.9)$
$52(3)$
$1.9(3.6)$
$60(38.7)$
$267(70)$
$124(37)$
$14.4(21)$

$28.0(10.6)$

58 (38.2)

7 (4.5)

2 (1.3)

82 (52.9)

32 (20.7)

11 (7.1)

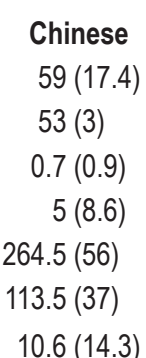

$10.6(14.3)$

23.3 (3.4)

$1(1.7)$

0 (0)

$1(1.7)$

28 (48.3)

$4(6.9)$

$5(8.5)$
$P$ value of comparisons

by Race/Ethnicity

$<0.0001$

0.11

$<0.0001$

$<0.0001$

0.0004

0.06

0.11

$<0.0001$

$<0.0001$

$<0.0001$

0.99

0.12

0.02

0.14

Table 2-Sleep characteristics of sample and by ethnic group

Median (IQR) unless noted as No. (\%)
Duration
PSG (min)
Self-report (h)
Continuity
Wake after sleep onset (min)
Percent sleep efficiency
Stage
Percent delta sleep
Sleep Disorder Breathing
Apnea-hypopnea index (AHI)
No. (\%) $\geq 15$ AHI
Desaturation event frequency
Percent total sleep $\leq 90 \%$ saturation
Self-report
Total Pittsburgh Sleep Quality Index (PSQI)
No. (\%) sleeping aids
No. (\%) medications affecting sleep

Total Sample

African

Americans

$387.0(71.1)$

$7.0(1.0)$

$44.3(34.3)$

$86.6(7.8)$

$1.8(4.6)$

$5.0(10.8)$

$66(20.4)$

$1.5(0.2)$

$0.1(1.1)$

5 (5.0)

53 (15.9)

94 (28.1)
$363.2(74.8)$

$6.0(2.0)$

$48.7(43.0)$

$83.1(11.3)$

$1.0(3.2)$

$6.0(11.3)$

$28(23.9)$

$2.0(6.4)$

$0.2(1.5)$

$6(5.0)$

$21(17.4)$

34 (28.1)
Caucasians

Chinese

393.1 (69.2)

$7.0(1.0)$

42.5 (30.9)

$87.1(6.7)$

$2.9(5.3)$

$5.0(9.6)$

28 (18.8)

$0.8(3.5)$

$0.1(1.7)$

5 (4.0)

22 (14.2)

47 (30.3)
$396.8(65.0)$

$7.0(1.8)$

$38.7(28.2)$

$88.5(7.0)$

$1.4(4.0)$

$4.2(13.0)$

$10(17.5)$

$1.4(4.6)$

$0(0.5)$

5 (4.0)

$10(17.2)$

$13(22.4)$
$P$ value of comparisons by Race/Ethnicity

$<0.0001$

0.0003

0.0005

$<0.0001$

$<0.0001$

0.30

0.49

0.04

0.0027

0.0002

0.73

0.52
DEF, percent TST $\leq 90 \%$ saturation), and poorer self-reported sleep quality in initial models (Table 3 ). Fully adjusted models showed that the sleep disorder-related measures were associated with higher CRP levels.

Higher fibrinogen levels were associated with less lower sleep efficiency, higher sleep disorder breathing measures (higher AHI, DEF, percent TST $\leq 90 \%$ saturation), and poorer self-reported sleep quality in initial models (Table 4). Like CRP, fully adjusted models showed that only the metrics of sleep disordered breathing were associated with higher fibrinogen levels.

Higher factor VIIc levels were associated with higher sleep disorder breathing-related measures in initial models (Table 5), but none remained significant in the fully adjusted models.
Higher PAI-1 levels were related to higher sleep disorder breathing-related measures in the initial and full models (Table 6).

\section{Ethnic Differences}

Significant interactions between ethnicity and sleep were found in the fully adjusted models for 3 sleep measures: sleep duration with CRP $(\mathrm{P}=0.01)$ and with PAI-1, $(\mathrm{P}=0.003)$; and WASO and sleep efficiency with fibrinogen $(\mathrm{P}=0.02$ and $\mathrm{P}=0.003$, respectively). Race stratified analyses showed that shorter sleep duration as measured by PSG was related to higher CRP levels among African Americans only $(\mathrm{P}=0.03)$, and to higher PAI-1 levels among Chinese $(\mathrm{P}=0.003)$. Greater WASO 
Table 3-Betas (standard errors) from regressions on sleep measures with C-reactive protein

\begin{tabular}{|c|c|c|}
\hline Sleep Measures & $\begin{array}{c}\text { Model } 1 \\
\text { Adjusted for age } \\
\text { and time duration } \\
\text { between measures }\end{array}$ & $\begin{array}{c}\text { Model } 2 \\
+ \text { all } \\
\text { covariates }\end{array}$ \\
\hline \multicolumn{3}{|l|}{ Duration } \\
\hline PSG (min) & $-5.74^{*}(2.50)$ & $-2.63+(2.92)$ \\
\hline Self-report (hours) & $-0.071(0.050)$ & $-0.0003(0.057)$ \\
\hline \multicolumn{3}{|l|}{ Continuity } \\
\hline Wake after sleep onset (log) & $0.052^{*}(0.025)$ & $-0.016(0.029)$ \\
\hline Efficiency & $-1.12^{\star * \star}(0.34)$ & $-0.178(0.394)$ \\
\hline \multicolumn{3}{|l|}{ Stage } \\
\hline Percent delta sleep & $0.104(0.190)$ & $0.267(0.215)$ \\
\hline \multicolumn{3}{|l|}{ Sleep Disorder Breathing } \\
\hline Apnea-hypopnea index (log) & $0.312^{* * * *}(0.059)$ & $0.344^{\star * * *}(0.065)$ \\
\hline Desaturation event frequency & $2.88^{* * * *}(0.51)$ & $2.57^{\star * \star *}(0.48)$ \\
\hline $\begin{array}{l}\text { Percent total sleep } \leq 90 \% \\
\text { saturation }\end{array}$ & $2.14^{* * * *}(0.52)$ & $1.76^{* * *}(0.47)$ \\
\hline \multicolumn{3}{|l|}{ Self-report } \\
\hline $\begin{array}{l}\text { Pittsburgh Sleep Quality } \\
\text { Inventory (PSQI:log) }\end{array}$ & $0.067^{* *}(0.023)$ & $-0.008(0.026)$ \\
\hline
\end{tabular}

Model 2 covariates include age, time duration between measures, site, ethnicity, menopausal status, body mass index (BMI), smoking status, medications affecting sleep, medications affecting hemostatic/inflammatory measures, and use of hormones. ${ }^{*} P<0.05$; ${ }^{* *} \mathrm{P}<0.01$; ${ }^{* * *} \mathrm{P}<0.001 ;{ }^{* * *} \mathrm{P}<0.0001 ;+$ indicates significant interaction with ethnicity in fully adjusted model.

$(\mathrm{P}=0.02)$ and lower sleep efficiency $(\mathrm{P}=0.009)$ were related to higher fibrinogen among African Americans only.

\section{DISCUSSION}

The present study evaluated the associations of sleep duration, continuity, depth, sleep disordered breathing, and sleep quality with biomarkers of inflammation and coagulation in a multi-ethnic sample of mid-life women. In initial models (adjusted for age and time between biomarkers and sleep study), we found a substantial number of associations. Consistent with other studies ${ }^{10,21}$ largely based on clinical populations, markers of sleep disordered breathing were positively correlated with CRP, fibrinogen, factor VIIc, and PAI-1. Less efficient sleep and poorer self-reported sleep quality were associated with CRP and fibrinogen levels. Shorter measured PSG and self-reported sleep duration were not associated with any biomarkers in the full sample. Thus, markers of sleep disordered breathing, sleep continuity, and quality were associated with biomarkers of inflammation and coagulation in the initial models.

Models that included additional covariates yielded a somewhat modified picture. Only the associations between biomarkers of inflammation and coagulation and indicators of sleep-disordered breathing remained statistically significant after full adjustment for medications that affect sleep or the biomarkers, use of hormone therapy, menopausal status, age, ethnicity, site, and residualized BMI. Each of the biomarkers, except factor VIIc, was associated with AHI, DEF, and/or percent TST $\leq 90 \%$ saturation. Although this pattern was not
Table 4-Betas (standard errors) from regressions on sleep measures with fibrinogen $(\mathrm{log})$ in full sample

\begin{tabular}{|c|c|c|}
\hline Sleep Measures & $\begin{array}{c}\text { Model } 1 \\
\text { Adjusted for age } \\
\text { and time duration } \\
\text { between measures }\end{array}$ & $\begin{array}{l}\text { Model } 2 \\
\quad+\text { all } \\
\text { covariates }\end{array}$ \\
\hline \multicolumn{3}{|l|}{ Duration } \\
\hline PSG (min) & $-24.42(15.99)$ & $-1.69(17.67)$ \\
\hline Self-report (h) & $-0.479(0.317)$ & $-0.051(0.344)$ \\
\hline \multicolumn{3}{|l|}{ Continuity } \\
\hline Wake after sleep onset (log) & $0.400 *(0.159)$ & $0.110+(0.176)$ \\
\hline Efficiency & $-7.34^{* *}(2.21)$ & $-2.85+(2.39)$ \\
\hline \multicolumn{3}{|l|}{ Stage } \\
\hline Percent delta sleep & $2.10(1.22)$ & $3.30^{*}(1.31)$ \\
\hline \multicolumn{3}{|l|}{ Sleep Disorder Breathing } \\
\hline Apnea-hypopnea index (log) & $1.21^{* *}(0.40)$ & $1.32^{* *}(0.39)$ \\
\hline Desaturation event frequency & $10.36^{* *}(3.42)$ & $9.70^{* * *}(2.88)$ \\
\hline $\begin{array}{l}\text { Percent total sleep } \leq 90 \% \\
\text { saturation }\end{array}$ & $10.12^{* *}(3.46)$ & $8.52^{* *}(2.88)$ \\
\hline \multicolumn{3}{|l|}{ Self-report } \\
\hline $\begin{array}{l}\text { Pittsburgh Sleep Quality } \\
\text { Inventory (log) }\end{array}$ & $0.45^{\star \star *}(0.16)$ & $0.12(0.17)$ \\
\hline
\end{tabular}

Model 2 covariates include age, time duration between measures, site, ethnicity, menopausal status, residualized body mass index, smoking status, medications affecting sleep, medications affecting hemostaticl inflammatory measures, and use of hormones. ${ }^{*} P<0.05$; ${ }^{* *} P<0.01$; ${ }^{* * *} \mathrm{P}<0.001 ;+$ indicates significant interaction with ethnicity in fully adjusted model.

unexpected ${ }^{22}$ it is noteworthy that these associations were obtained in mid-life women, from community samples with no diagnosed sleep disorders, based on in-home sleep assessments, and adjusted for the many covariates that impact sleep or the biomarkers.

Ethnic differences in the patterns with sleep duration and efficiency were apparent. Shorter PSG sleep duration was associated with higher levels of CRP in African Americans and with higher levels of PAI-1 in Chinese. Less efficient sleep and greater WASO were associated with higher levels of fibrinogen in African Americans. Although these analyses were exploratory and represent an initial effort to evaluate ethnic variation in associations, the consistency of effects showing that African Americans who have elevated inflammatory markers are at higher risk for short and discontinuous sleep is worthy of further investigation. These ethnic variations may be affected by differences in adiposity (including proportion of subcutaneous and visceral fat) by history and current status, which are beyond the scope of our analysis. Furthermore, these effects suggest that products in the inflammation and coagulation pathways are important for their involvement in the link between sleep and cardiometabolic health in African Americans, whereas sleep disordered breathing indicators may be important pathways for women's cardiometabolic health, regardless of ethnicity.

Self-reported usual sleep duration was not associated with any of the studied biomarkers. The null results are not consistent with experimentally-induced sleep restriction studies, which show that whether inflammation increases or decreases is 
Table 5-Betas (standard errors) from regressions on sleep measures with factor VII-c (log) in full sample

\begin{tabular}{|c|c|c|}
\hline Sleep Measures & $\begin{array}{c}\text { Model } 1 \\
\text { Adjusted for age } \\
\text { and time duration } \\
\text { between measures }\end{array}$ & $\begin{array}{c}\text { Model } 2 \\
+ \text { all } \\
\text { covariates }\end{array}$ \\
\hline \multicolumn{3}{|l|}{ Duration } \\
\hline PSG (min) & $-8.54(12.36)$ & $-15.29(12.76)$ \\
\hline Self-report (hours) & $-0.076(0.245)$ & $0.0008(0.2477)$ \\
\hline \multicolumn{3}{|l|}{ Continuity } \\
\hline Wake after sleep onset (log) & $-0.103(0.124)$ & $-0.026(0.128)$ \\
\hline Efficiency & $1.72(1.73)$ & $0.507(1.74)$ \\
\hline \multicolumn{3}{|l|}{ Stage } \\
\hline Percent delta sleep & $-0.114(0.947)$ & $-0.611(0.958)$ \\
\hline \multicolumn{3}{|l|}{ Sleep Disorder Breathing } \\
\hline Apnea-hypopnea index (log) & $0.827^{\star *}(0.313)$ & $0.502(0.304)$ \\
\hline Desaturation event frequency & $9.21^{* * *}(2.73)$ & $4.55^{\star}(2.22)$ \\
\hline $\begin{array}{l}\text { Percent total sleep } \leq 90 \% \\
\text { saturation }\end{array}$ & $5.86^{*}(2.79)$ & $3.65(2.22)$ \\
\hline \multicolumn{3}{|l|}{ Self-report } \\
\hline $\begin{array}{l}\text { Pittsburgh Sleep Quality -log } \\
\text { scale }\end{array}$ & $0.15(0.12)$ & $0.096(0.124)$ \\
\hline
\end{tabular}

Model 2 includes covariates: age, time duration between measures, site, ethnicity, menopausal status, residualized body mass index, smoking status, medications affecting sleep, medications affecting hemostaticl inflammatory measures, and use of hormones. ${ }^{*} P<0.05$; ${ }^{* *} P<0.01$; ${ }^{* * *} P<0.001$.

related to the length of sleep restriction. ${ }^{23-26}$ However, our negative findings are similar to results from other epidemiological studies showing inconsistent relationships with short sleep. ${ }^{27-29}$ Categorization of short sleep (usually self-report) varies widely in various epidemiological studies, ranging from 4 hours or less to 7 hours or less. ${ }^{30,31}$ In our sample, only $8.2 \%$ actually slept 5 hours or less, $24.7 \%$ slept between 5 and 6 hours, $43.5 \%$ slept between 6 and 7 hours, $20.3 \%$ slept 7 to 8 hours, and $3.3 \%$ slept $>8$ hours. It is possible that there was insufficient variability of sleep duration in our sample to detect an association. It is also possible that usual sleep duration is unrelated in community studies (as opposed to experimental studies) and subsets of individuals with short sleep are vulnerable to increases in inflammation, as we report herein for African American women.

Limitations of our study include the cross-sectional nature of the design; the limited range of sleep duration in our sample; the exclusion of some important biomarkers of inflammation and coagulation, e.g., IL-6, TNF- $\alpha$, and von Willebrand factor. In addition, the nature of the study population, focusing solely on women, precluded examination of gender differences. Along these lines, some data suggest different patterns with self-reported sleep characteristics in men and women. ${ }^{29,32}$ Strengths are the multiethnic nature of the community sample, and 3 nights of in-home PSG studies. The large, gender-homogeneous sample permits greater power to assess the relations in mid-life women.

In conclusion, our study found that women with sleep disordered breathing had elevated levels of inflammatory and coagulation biomarkers. Moreover, African Americans who had short and inefficient sleep had elevated CRP and fibrinogen levels.
Table 6-Betas (standard errors) from regressions on sleep measures with PAl-1 (log) in full sample

\begin{tabular}{|c|c|c|}
\hline Sleep Measures & $\begin{array}{c}\text { Model } 1 \\
\text { Adjusted for age } \\
\text { and time duration } \\
\text { between measures }\end{array}$ & $\begin{array}{l}\text { Model } 2 \\
\quad+\text { all } \\
\text { covariates }\end{array}$ \\
\hline \multicolumn{3}{|l|}{ Duration } \\
\hline PSG (min) & $-5.73(3.33)$ & $-4.75+(3.49)$ \\
\hline Self-report (h) & $-0.0202(0.0665)$ & $0.0076(0.068)$ \\
\hline \multicolumn{3}{|l|}{ Continuity } \\
\hline Wake after sleep onset (log) & $0.02(0.03)$ & $-0.012(0.03)$ \\
\hline Efficiency & $-1.05^{*}(0.46)$ & $-0.58(0.47)$ \\
\hline \multicolumn{3}{|l|}{ Stage } \\
\hline Percent delta sleep & $0.17(0.25)$ & $0.23(0.26)$ \\
\hline \multicolumn{3}{|l|}{ Sleep Disorder Related } \\
\hline Apnea-hypopnea index (log) & $0.24^{\star *}(0.08)$ & $0.28^{* * *}(0.08)$ \\
\hline Desaturation event frequency & $1.90^{* *}(0.70)$ & $2.04^{* * *}(0.57)$ \\
\hline $\begin{array}{l}\text { Percent total sleep } \leq 90 \% \\
\text { saturation }\end{array}$ & $1.72^{*}(0.70)$ & $1.61^{* *}(0.56)$ \\
\hline \multicolumn{3}{|l|}{ Self-report } \\
\hline $\begin{array}{l}\text { Pittsburgh Sleep Quality -log } \\
\text { scale }\end{array}$ & $0.06(0.03)$ & $0.02(0.03)$ \\
\hline
\end{tabular}

Model 2 includes covariates: age, time duration between measures, site, ethnicity, menopausal status, residualized body mass index, smoking status, medications affecting sleep, medications affecting hemostaticl inflammatory measures, and use of hormones. ${ }^{*} P<0.05$; ${ }^{* *} P<0.01$; ${ }^{* * *} \mathrm{P}<0.001 ;+$ indicates significant interaction with ethnicity in fully adjusted model.

These findings suggest that inflammation and coagulation may provide a pathway connecting these sleep characteristics with risk for cardiometabolic disorders. However, our study only examined associations and the direction of the effects may be reversed from that posited. Longitudinal studies with multiple measures are needed to identify the temporal relationships between sleep characteristics and inflammation.

\section{ACKNOWLEDGMENTS}

Funding for the Study of Women's Health Across the Nation (SWAN) Sleep Study is from the National Institute on Aging (Grants AG019360, AG019361, AG019362, AG019363). The SWAN has grant support from the National Institutes of Health (NIH), DHHS, through the National Institute on Aging (NIA), the National Institute of Nursing Research (NINR) and the NIH Office of Research on Women's Health (ORWH) (Grants NR004061; AG012505, AG012535, AG012531, AG012539, AG012546, AG012553, AG012554, AG012495). The content of this article is solely the responsibility of the authors and does not necessarily represent the official views of the NIA, NINR, ORWH, or the NIH.

Clinical Centers: University of Michigan, Ann Arbor MaryFran Sowers, PI; Massachusetts General Hospital, Boston, MA - Joel Finkelstein, PI 1999 - present; Robert Neer, PI 1994 - 1999; Rush University, Rush University Medical Center, Chicago, IL - Howard Kravitz, PI 2009 - present; Lynda Powell, PI 1994 - 2009; University of California, Davis/Kaiser - Ellen Gold, PI; University of California, Los Angeles - Gail 
Greendale, PI; Albert Einstein College of Medicine, Bronx, NY - Rachel Wildman, PI 2010; Nanette Santoro, PI 2004 - 2010; University of Medicine and Dentistry - New Jersey Medical School, Newark - Gerson Weiss, PI 1994 - 2004; and the University of Pittsburgh, Pittsburgh, PA - Karen Matthews, PI.

NIH Program Office: National Institute on Aging, Bethesda, MD - Sherry Sherman 1994 - present; Marcia Ory 1994 - 2001; National Institute of Nursing Research, Bethesda, MD - Project Officer.

Central Laboratory: University of Michigan, Ann Arbor Daniel McConnell (Central Ligand Assay Satellite Services).

Coordinating Center: University of Pittsburgh, Pittsburgh, PA - Kim Sutton-Tyrrell, PI 2001 - present; New England Research Institutes, Watertown, MA - Sonja McKinlay, PI 1995 -2001 .

Steering Committee: Susan Johnson, Current Chair; Chris Gallagher, Former Chair

We thank the study staff at each site and all the women who participated in SWAN.

\section{DISCLOSURE STATEMENT}

This was not an industry supported study. Dr. Buysse has consulted for Actelion, Arena, Cephalon, Eli Lilly, GlaxoSmithKline, Merck, Neurocrine, Neurogen, Pfizer, Respironics, Sanofi-Aventis, Sepracor, Servier, Somnus, Stress Eraser, Takeda, and Transcept; has received research support from Sepracor; and has helped to produce CME materials and has given paid CME lectures indirectly supported by industry sponsors. Dr. Sanders has consulted for Philips-Respironics. $\mathrm{He}$ is a co-inventor of $\mathrm{BiPAP} \AA$ manufactured by PhilipsRespironics and has a financial interest in this brand and related technologies by Philips-Respironics. The other authors have indicated no financial conflicts of interest.

\section{REFERENCES}

1. Gallicchio L, Kalesan B. Sleep duration and mortality: a systematic review and meta-analysis. J Sleep Res 2009;18:148-58.

2. Ayas NT, White DP, Al-Delaimy WK, et al. A prospective study of selfreported sleep duration and incident diabetes in women. Diabetes Care $2003 ; 26: 380-4$

3. Shamsuzzaman AS, Gersh BJ, Somers VK. Obstructive sleep apnea: implications for cardiac and vascular disease. JAMA 2003;290:1906-14.

4. Youngstedt S, Kripke DF. Long sleep and mortality: rationale for sleep restriction. Sleep Med Rev 2004;8:159-74.

5. Patel SR, Hu FB. Short sleep duration and weight gain: a systematic review. Obesity (Silver Spring) 2008;16:643-53.

6. De Taeye B, Smith LH, Vaughan DE. Plasminogen activator inhibitor-1: a common denominator in obesity, diabetes and cardiovascular disease. Curr Opin Pharmacol 2005;5:149-54.

7. Folsom AR. Hemostatic risk factors for atherothrombotic disease: an epidemiologic view. Thromb Haemost 2001;86:366-73.

8. Shah K. Thrombogenic risk factors for atherothrombosis. Rev Cardiovasc Med 2006;7:10-6.

9. Simpson N, Dinges DF. Sleep and inflammation. Nutr Rev 2007;65:S244S252.

10. McNichols WT, Bonsignore MR, on behalf of the Management Committee of EU COST ACTION B26. Sleep apnoea as an independent risk factor for cardiovascular disease: current evidence, basic mechanisms and research priorities. Eur Respir J 2007;29:156-78.
11. Ross R. Atherosclerosis--an inflammatory disease. N Engl J Med 1999;340:115-26.

12. Danesh J, Collins R, Appleby P, Peto R. Association of fibrinogen, Creactive protein, albumin, or leukocyte count with coronary heart disease: meta-analyses of prospective studies. JAMA 1998;279:1477-82.

13. Danesh J, Whincup P, Walker M, et al. Fibrin D-dimer and coronary heart disease: prospective study and meta-analysis. Circulation 2001;103:2323-7.

14. Tracy RP. Thrombin, inflammation, and cardiovascular disease: an epidemiologic perspective. Chest 2003;124:49S-57S.

15. Hall M, Matthews KA, Kravitz H, et al. Race and financial strain are independent correlates of sleep in mid-life women: The SWAN Sleep Study. Sleep 2009;32:72-82.

16. Matthews KA, Sowers MF, Derby CA, et al. Ethnic differences in cardiovascular risk factor burden among middle-aged women: Study of Women's Health Across the Nation (SWAN). Am Heart J 2005;149:1066-73.

17. Sowers M, Crawford S, Sternfeld B, et al. SWAN: a multi-center, multiethnic community-based cohort study of women and the menopausal transition. In: Lobo R, Marcus R, Kelsey J, eds. Menopause: biology and pathology. New York: Academic Press, 2000.

18. Buysse DJ, Reynolds CF III, Monk TH, Berman SR, Kupfer DJ. The Pittsburgh Sleep Quality Index: a new instrument for psychiatric practice and research. Psychiatry Res 1989;28:193-213.

19. Sleep-related breathing disorders in adults: recommendations for syndrome definition and measurement techniques in clinical research. The Report of an American Academy of Sleep Medicine Task Force. Sleep 1999;22:667-89.

20. Rechtshaffen A, Kales A. A manual of standardized terminology, techniques, and scoring systen for sleep stages of human subjects (NIH publication 204). Washington, DC: U.S. Government Printing Office, Department of Health Education and Welfare, 1968.

21. Robinson GV, Pepperell JC, Segal HC, Davies RJ, Stradling JR. Circulating cardiovascular risk factors in obstructive sleep apnoea: data from randomised controlled trials. Thorax 2004;59:777-82.

22. von Kanel R, Loredo JS, Ancoli-Israel S, Mills PJ, Natarajan L, Dimsdale JE. Association between polysomnographic measures of disrupted sleep and prothrombotic factors. Sleep Med 2007;131:733-9.

23. Haack M. Elevated inflammatory markers in response to prolonged sleep restriction are associated with increased pain experience in healthy volunteers. Sleep 2007;30:1145-52.

24. Meier-Ewert HK, Ridker PM, Rifai N, et al. Effect of sleep loss on C-reactive protein, an inflammatory marker of cardiovascular risk. J Am Coll Cardiol 2004;43:678-83.

25. van Leeuwen WM, Lehto M, Karisola P, et al. Sleep restriction increases the risk of developing cardiovascular diseases by augmenting proinflammatory responses through IL-17 and CRP. PLoS One 2009;4:e4589.

26. Frey DJ, Fleshner M, Wright KP Jr. The effects of 40 hours of total sleep deprivation on inflammatory markers in healthy young adults. Brain Behav Immunol 2007;21:1050-7.

27. Taheri S, Austin D, Lin L, Nieto FJ, Young T, Mignot E. Correlates of serum C-reactive protein (CRP)--no association with sleep duration or sleep disordered breathing. Sleep 2007;30:991-6.

28. Patel SR, Zhu X, Storfer-Isser A, et al. Sleep duration and biomarkers of inflammation. Sleep 2009;32:200-4.

29. Miller MA, Kandala NB, Kivimaki M, et al. Gender differences in the cross-sectional relationships between sleep duration and markers of inflammation: Whitehall II study. Sleep 2009;32:857-64.

30. Heslop P, Smith GD, Metcalfe C, Macleod J, Hart C. Sleep duration and mortality: the effect of short or long sleep duration on cardiovascular and all-cause mortality in working men and women. Sleep Med 2002;3:305-14.

31. Tamakoshi A, Ohno Y. Self-reported sleep duration as a predictor of allcause mortality: results from the JACC Study, Japan. Sleep 2004;27:51-4.

32. Suarez EC. Self-reported symptoms of sleep disturbance and inflammation, coagulation, insulin resistance and psychosocial distress: evidence for gender disparity. Brain Behav Immunol 2008;22:960-8. 